\title{
Lifelong genetically lowered sclerostin and risk of cardiovascular disease
}

Jonas Bovijn, $\mathrm{MD}^{1,2^{*}}$; Kristi Krebs, $\mathrm{MSc}^{3^{*}}$; Chia-Yen Chen, $\mathrm{PhD}^{4-6^{*}}$; Ruth Boxall, $\mathrm{MSc}^{7,8 *}$; Jenny C. Censin, MD ${ }^{1,2}$; Teresa Ferreira, $\mathrm{PhD}^{1}$; Sara L. Pulit, PhD ${ }^{1,9,10}$; Craig A. Glastonbury, $\mathrm{PhD}^{1}$; Samantha Laber, DPhil1,2; lona Y. Millwood, DPhil7,8; Kuang Lin, PhD77 Liming Li, MD ${ }^{11}$; Zhengming Chen, DPhil7; Lili Milani, $\mathrm{PhD}^{3}$; Robin G. Walters, $\mathrm{PhD}^{7,8^{*}}$; Reedik Mägi, $\mathrm{PhD}^{3^{*}}$; Benjamin M. Neale, $\mathrm{PhD}^{4,6^{*}}$; Cecilia M. Lindgren, $\mathrm{PhD}^{1,2,9,12^{*}}$; Michael V. Holmes, MD PhD ${ }^{1,7,8,12^{*}}$

\section{Affiliations:}

1. Big Data Institute at the Li Ka Shing Centre for Health Information and Discovery, University of Oxford, Oxford, OX3 7FZ, United Kingdom.

2. Wellcome Centre for Human Genetics, Nuffield Department of Medicine, University of Oxford, Oxford, OX3 7BN, United Kingdom.

3. Estonian Genome Center, Institute of Genomics, University of Tartu, Tartu, Estonia.

4. Analytic and Translational Genetics Unit, Massachusetts General Hospital, Boston, MA, USA.

5. Psychiatric and Neurodevelopmental Genetics Unit, Boston, MA, USA.

6. Stanley Center for Psychiatric Research, Broad Institute of MIT and Harvard, Cambridge, MA, USA.

7. Clinical Trial Service Unit and Epidemiological Studies Unit (CTSU), Nuffield Department of Population Health, University of Oxford, Oxford, OX3 7LF, United Kingdom.

8. Medical Research Council Population Health Research Unit (MRC PHRU), Nuffield Department of Population Health, University of Oxford, Oxford, OX3 7LF, United Kingdom.

9. Program in Medical and Population Genetics, Broad Institute, Cambridge, MA, USA.

10. Department of Genetics, University Medical Center Utrecht, Utrecht, The Netherlands.

11. Department of Epidemiology \& Biostatistics, Peking University Health Science Centre, Peking University, Beijing, China.

12. National Institute for Health Research Oxford Biomedical Research Centre, Oxford University Hospitals NHS Foundation Trust, John Radcliffe Hospital, Oxford, United Kingdom. 
* These authors contributed equally

\section{Corresponding authors:}

Name: Dr Jonas Bovijn, MD MSc

Address: Big Data Institute at the Li Ka Shing Centre for Health Information and Discovery, University of Oxford, Oxford, OX3 7FZ, United Kingdom.

Email: jbovijn@well.ox.ac.uk

Name: Professor Cecilia M Lindgren, PhD

Address: Big Data Institute at the Li Ka Shing Centre for Health Information and Discovery, University of Oxford, Oxford, OX3 7FZ, United Kingdom.

Email: celi@broadinstitute.org

Name: Associate Professor Michael V Holmes, MD PhD

Address: Medical Research Council Population Health Research Unit (MRC PHRU), Nuffield Department of Population Health, University of Oxford, Old Road Campus, Oxford, OX3 7LF, United Kingdom.

Email: michael.holmes@ndph.ox.ac.uk 


\section{Abstract}

\section{Background}

Inhibition of sclerostin is a novel therapeutic approach to lowering fracture risk. However, phase III randomised controlled trials (RCTs) of romosozumab, a monoclonal antibody that inhibits sclerostin, suggest an imbalance of serious cardiovascular events.

\section{Methods}

We used two independent genetic variants (rs7209826 and rs188810925) in SOST (encoding sclerostin) associated with bone mineral density (BMD) as proxies for therapeutic inhibition of sclerostin. We estimated the effects on risk of osteoporosis, fracture, coronary heart disease (CHD) and a further 22 cardiometabolic risk factors and diseases, by combining data from up to 478,967 participants of European ancestry from three prospective cohorts and up to $1,030,836$ participants from nine GWAS consortia. In addition, we performed meta-analyses of cardiovascular outcome data from phase III RCTs of romosozumab.

\section{Results}

Meta-analysis of RCTs identified a higher risk of cardiac ischemic events in patients randomised to romosozumab (25 events among 4,298 individuals; odds ratio [OR] 2.98; 95\% confidence interval [Cl], $1 \cdot 18$ to $7.55 ; P=0.017)$. Scaled to the equivalent dose of romosozumab $\left(210 \mathrm{mg} / \mathrm{month} ; 0.09 \mathrm{~g} / \mathrm{cm}^{2}\right.$ higher $\left.\mathrm{BMD}\right)$, the SOST variants associated with lower risk of fracture (OR, 0.59;95\% $\left.\mathrm{Cl}, 0.54-0.66 ; \mathrm{P}=1.4 \times 10^{-24}\right)$, and osteoporosis (OR, $\left.0.43 ; 95 \% \mathrm{Cl}, 0.36-0.52 ; \mathrm{P}=2 \cdot 4 \times 10^{-18}\right)$. The SOST variants associated with higher risk of myocardial infarction and/or coronary revascularisation (69,649 cases; OR, 1.18; 95\% Cl, 1.06-1.32; $\mathrm{P}=0.003)$ and type 2 diabetes (OR 1.15;95\% Cl, 1.05-1.27; $\mathrm{P}=0.003)$, higher systolic blood pressure $\left(1.3 \mathrm{mmHg} ; 95 \% \mathrm{Cl} 0 \cdot 8-1.9 ; \mathrm{P}=5 \cdot 9 \times 10^{-6}\right)$ and waist-to-hip-ratio adjusted for $\mathrm{BMI}(0.05 \mathrm{SDs} ; 95 \% \mathrm{Cl}, 0.02$ to $\left.0.08 ; P=8.5 \times 10^{-4}\right)$. 
bioRxiv preprint doi: https://doi.org/10.1101/531004; this version posted February 1,2019 . The copyright holder for this preprint (which was not certified by peer review) is the author/funder, who has granted bioRxiv a license to display the preprint in perpetuity. It is made available under aCC-BY 4.0 International license.

\section{Conclusions}

Genetically and therapeutically lowered sclerostin leads to higher risk of cardiovascular events. Rigorous evaluation of the cardiovascular safety of romosozumab and other sclerostin inhibitors is warranted. 


\section{Introduction}

Osteoporosis, a disorder of bone demineralisation, is a common condition associated with considerable morbidity and mortality, particularly among postmenopausal women and the elderly. ${ }^{1} \mathrm{~A}$ range of therapeutics for osteoporosis is currently available, though these agents are plagued by poor adherence (fewer than $40 \%$ of patients prescribed oral bisphosphonates still take these medications after one year ${ }^{2}$ ), occurrence of rare but serious adverse events (e.g., bisphosphonate-induced osteonecrosis of the jaw), high cost, and uncertainty regarding long-term efficacy. ${ }^{3}$ There is therefore a substantial demand for effective, safe and well-tolerated anti-osteoporotic therapies. ${ }^{4}$

Sclerostin, a glycoprotein encoded by the SOST gene, is a negative regulator of bone formation that is secreted by osteocytes. It inhibits Wnt signaling, which leads to down-regulation of osteoblast development and function. ${ }^{5}$ Loss-of-function mutations in SOST lead to sclerosteosis, a rare autosomal recessive condition characterised by bone overgrowth. ${ }^{6}$ Similarly, van Buchem's disease, another rare autosomal recessive condition with a clinical picture similar to sclerosteosis (albeit generally milder), is caused by a deletion of a SOST-specific regulatory element. ${ }^{7}$ The discovery of functional variants in SOST as the underlying cause of these rare conditions of bone overgrowth nearly 2 decades ago, led to the development of sclerostin inhibitors as a treatment for osteoporosis. ${ }^{8}$

Three anti-sclerostin monoclonal antibodies have been, or are, currently in clinical development, ${ }^{5}$ including romosozumab (Amgen, UCB) and blosozumab (Eli Lilly and Company) for osteoporosis and setrusumab (Mereo BioPharma), currently in phase Ilb for osteogenesis imperfecta. Despite phase II results showing significant improvements in bone mineral density (BMD), ${ }^{9}$ a biomarker used to evaluate the effect of antiosteoporotic treatments, clinical development for blosozumab was halted in 2015 , reportedly due to injection site reactions. ${ }^{10}$ Phase II and III randomised controlled trials (RCTs) have shown that romosozumab is effective at increasing BMD in both men and women, whilst also reducing vertebral and non-vertebral fracture risk in women. ${ }^{11-14}$ However, adverse event data reported in the phase III BRIDGE and $\mathrm{ARCH}$ trials (in men and post-menopausal women, respectively) have suggested that romosozumab 
may be associated with an excess risk of cardiovascular events. ${ }^{13,14}$ Concerns about the cardiovascular safety of romosozumab, and sclerostin-inhibition more generally, have previously been raised. ${ }^{8,15-17}$

In January 2019, the Japanese Ministry of Health, Labor and Welfare approved the use of romosozumab in men and postmenopausal women with osteoporosis at high risk of fracture. ${ }^{18}$ The US Food and Drug Administration (FDA) and the European Medicines Agency (EMA) are currently considering licensing applications for romosozumab for the treatment of osteoporosis in men and postmenopausal women at increased risk of fracture..$^{19,20}$ A detailed appraisal of the cardiovascular effects of sclerostin inhibition by exploiting randomised data from orthogonal sources is both timely and warranted, and may assist in the evaluation of whether this class of drugs represents a rational and safe therapeutic strategy for the prevention of fracture.

Naturally-occurring human genetic variation can serve as a proxy for therapeutic stimulation or inhibition of a drug target, presenting a valuable opportunity to assess the likely consequences of modifying a therapeutic target on both the intended therapeutic effects and target-mediated adverse drug reactions. ${ }^{21}$ The application of this approach in a Mendelian randomisation framework has previously shown to recapitulate known clinical effects of drug target modulation. ${ }^{22-26}$ In this study, we used this genetic approach to examine the effect of BMD-increasing alleles in the SOST locus (as a proxy for sclerostin inhibition) on the risk of bone fracture, osteoporosis, cardiovascular risk factors and disease, to shed light on whether treatment with sclerostin inhibitors is likely to adversely impact on cardiovascular disease. 


\section{Methods}

\section{Study population}

We examined individual-level genotypic and phenotypic data for 502,617 subjects in UK Biobank (UKBB), a population-based cohort based in the United Kingdom. Following quality control, 423,761 subjects of white ethnicity were included for further analysis (see Supplementary Methods and Table S1 in the appendix for full quality control descriptions and baseline characteristics of participants in UKBB). In addition, we included data from a further two European-ancestry cohorts: Partners HealthCare Biobank (PHB; up to 19,132 subjects) and Estonian Biobank (EGCUT; up to 36,074 subjects). Trans-ethnic replication was attempted in China Kadoorie Biobank (CKB; up to 81,546 subjects). See Supplementary Methods in the appendix for further detail on each cohort.

We supplemented these data with summary-level data from 9 genome-wide association study (GWAS) consortia, including data for BMD (ultrasound-derived estimated heel-bone BMD [up to 142,487 individuals] and dual-energy x-ray absorptiometry (DXA)-derived BMD [up to 49,988]), coronary heart disease (CHD; up to 60,801 cases and 123,504 controls) and myocardial infarction, stroke (up to 40,585 cases and 406,111 controls), atrial fibrillation (up to 60,620 cases and 970,216 controls), type 2 diabetes mellitus (T2D; up to 74,124 cases and 824,006 controls), glycemic traits (up to 123,665 individuals), serum lipid fractions (up to 92,804 individuals), anthropometric traits (up to 222,233 individuals), and renal function (up to 110,515 individuals). Further details on each consortium are provided in the Supplementary Methods and Table $\mathbf{S} 2$ in the appendix. An overview of the overall study design is shown in Figure $\mathbf{S} 1$ in the appendix. All studies contributing data to these analyses were approved by their local ethics committees (details in appendix).

\section{Genetic instrument selection and validation}

A recent large-scale GWAS for estimated heel-bone BMD (eBMD), conducted in 142,487 individuals, ${ }^{27}$ identified two conditionally independent $\left(r^{2}=0.13\right.$ among European ancestry individuals in UKBB) genetic variants in the SOST locus associated with eBMD: rs7209826 ( $\mathrm{A}>\mathrm{G}, \mathrm{G}$-allele frequency in UKBB $=40 \%$ ) and $\mathrm{rs} 188810925(\mathrm{G}>\mathrm{A}, \mathrm{A}$-allele frequency $=8 \%$ ). Both SNPs are located $\sim 35 \mathrm{~kb}$ downstream from SOST 
and fall within or near a $52 \mathrm{~kb}$ area that contains the van Buchem disease deletion (Figure S2 in the appendix), a region previously shown to affect SOST expression in human bone. ${ }^{7}$ Recent functional evidence has also shown that SNPs in this area (one of which, $r s 7220711$, is in high LD $\left[r^{2}=0.99\right]$ with rs7209826) regulate SOST expression via differential transcription factor binding. ${ }^{28}$ Previous Mendelian randomisation studies have also made use of non-coding variants with an effect on gene expression as proxies for pharmacologic modulation of the same target-gene..$^{22,26,29,30}$

We extracted estimates for these two SNPs (rs7209826 and rs188810925) from GWAS consortia listed in Table S2 in the appendix. For GWAS datasets that did not include these variants, we selected variants in high LD ( $\left.r^{2}>0.9\right)$ with our selected SNPs (See Supplementary Methods in the appendix for details). We selected rs7220711 as a proxy for rs7209826 based on high LD ( $r^{2}=0.99$ in European ancestry populations), availability across most consortia, and prior functional evidence linking rs7220711 to SOST expression (see above). In addition, we validated the effect of rs7220711 on various measures of BMD as being comparable to that of rs7209826 (Figure S3 in the appendix). There were no suitable proxies $\left(r^{2}>0 \cdot 9\right)$ for $r s 188810925$.

We examined the associations of rs7209826 and rs188810925 (and their selected proxies) on DXA-derived measures of BMD measured at specific body sites (lumbar spine, femoral neck, and forearm), using data from the largest GWAS to date for these phenotypes. ${ }^{31}$

We next examined the effect of these variants on SOST mRNA expression levels in various human tissues in the Genotype-Tissue Expression (GTEx) project dataset. ${ }^{32}$

\section{Study outcomes}

Detailed definitions of each outcome studied are described in the Supplementary Methods and Tables S3-5 in the appendix. We first tested the association of rs7209826 and rs188810925 with key efficacy outcomes, i.e., fracture risk and risk of osteoporosis (both defined as a combination of self-reported outcomes and International Classification of Diseases, ninth and tenth revision (ICD-9 and ICD-10) codes). 
Next, we examined the association of the BMD-increasing alleles of rs7209826 and rs188810925 with risk of myocardial infarction and/or coronary revascularisation (including self-reported and ICD-9/ICD-10 codes for myocardial infarction, coronary artery bypass graft surgery and/or percutaneous transluminal coronary angioplasty) and a broader composite of all coronary heart disease (CHD; including all codes for myocardial infarction [MI] and/or coronary revascularisation, plus self-reported and ICD-9/ICD-10 codes for angina and chronic stable ischemic heart disease; see Tables S3-5 in the appendix for specific codes included). Secondary disease outcomes included additional cardiovascular outcomes of interest (ischemic stroke, hemorrhagic stroke, all stroke, peripheral vascular disease, atrial fibrillation, heart failure), chronic kidney disease, aortic aneurysm and aortic stenosis (given sclerostin's putative role in these conditions ${ }^{17,33,34}$ ), hypertension and T2D. Association with 11 quantitative cardiometabolic traits, many of which are established as causal risk factors for CHD, were also evaluated (systolic blood pressure (SBP), diastolic blood pressure (DBP), body mass index (BMI), waist-hip ratio (WHR) adjusted for BMI, low-density lipoprotein (LDL) cholesterol, high-density lipoprotein (HDL) cholesterol, triglycerides, fasting glucose, fasting insulin, $\mathrm{HbA} 1 \mathrm{c}$ and serum creatinine-estimated glomerular filtration rate (eGFR)).

\section{Statistical Analyses}

We derived cohort-specific SNP effect estimates from participants in the 4 prospective cohorts (see Supplementary Methods for specific methodology applied in each cohort). Estimates $(\log (\mathrm{OR})$ and the standard error of $\log (\mathrm{OR})$ for binary outcomes and beta and the standard error of beta for quantitative traits) for the per-allele effect of these variants on disease outcomes and quantitative traits were aligned to the BMD-increasing alleles (as per the effect of therapeutic sclerostin inhibition), and scaled to an increase in BMD equivalent to that reported with romosozumab treatment (see below). We then meta-analysed estimates from the prospective cohorts with (scaled) estimates from GWAS consortia for equivalent outcomes using inverse-variance weighted fixed-effect meta-analysis. We predefined a $p$-value threshold of $<0.05$ for the association with CHD given the reported association of sclerostin inhibition with cardiac ischemic events in prior RCTs. ${ }^{13,14}$ For the remaining 11 cardiometabolic outcomes and 11 quantitative traits, we set a Bonferroni adjusted p-value threshold of $<0 \cdot 0045(0 \cdot 05 / 11)$. 


\section{Scaling of allelic estimates}

We scaled allelic estimates pertaining to risk of osteoporosis, fractures, cardiometabolic outcomes and quantitative traits to an increase in BMD equivalent to that reported in a phase II RCT of 12 months of $210 \mathrm{mg}$ romosozumab monthly. ${ }^{35}$ This corresponds to the dose evaluated in phase III RCTs of romosozumab, and represents a $0.09 \mathrm{~g} / \mathrm{cm}^{2}$ increase in lumbar spine BMD (LS-BMD) in postmenopausal women. ${ }^{35}$ See Supplementary Methods in the appendix for further details.

\section{Meta-analysis of cardiovascular outcomes in RCTs of sclerostin-inhibitors}

We searched for all phase III RCTs performed for sclerostin inhibitors (see Supplementary Methods in the appendix for further details). We then performed meta-analyses of cardiovascular outcome data at 12 months from phase III RCTs using $210 \mathrm{mg}$ romosozumab monthly for 12 months, with 'cardiac ischemic events' as the primary outcome of interest. Further meta-analyses were performed for 'cerebrovascular events' and a composite outcome of 'serious cardiovascular events' (including cardiac ischemic events, cerebrovascular events, heart failure, cardiovascular death, non-coronary revascularisation and peripheral vascular ischemic events not requiring revascularisation). We set a prespecified $p$-value threshold of $<0 \cdot 05$ for meta-analyses of the RCTs given the prior evidence for ischemic cardiovascular events seen in individual RCTs of romosozumab. ${ }^{13,14}$

All meta-analyses of RCT cardiovascular outcomes were performed according to the Mantel-Haenszel method without continuity correction, ${ }^{36,37}$ a non-parametric test designed for rare outcomes. Additional sensitivity analyses were performed using the Peto method ${ }^{37}$ (see Supplementary Methods in the appendix).

\section{Role of the funding source}

The funders of the study had no role in study design, data collection, data analysis, data interpretation, or writing of the report. The corresponding authors (JB, CML and MVH) had full access to all the data in the study and shared final responsibility for the decision to submit for publication with all authors. 


\section{Results}

\section{Risk of cardiovascular events in phase III RCTs of sclerostin inhibitors}

Romosozumab was the only sclerostin inhibitor with data from phase III RCTs. Four phase III RCTs of romosozumab, including 11,954 individuals were identified (Table $\mathbf{S 6}$ in the appendix), of which three trials (BRIDGE ${ }^{14}, \mathrm{ARCH}^{13}$ and $\mathrm{FRAME}^{12}$ ) reported cardiovascular adverse event data (Table S7 in the appendix). Only the $\mathrm{BRIDGE}^{14}$ and $\mathrm{ARCH}^{13}$ trials reported data on cardiac ischemic events and cerebrovascular events.

Meta-analysis of 25 cardiac ischemic events in 4,298 individuals, from two RCTs (BRIDGE ${ }^{14}$ and ARCH ${ }^{13}$ ) identified that $210 \mathrm{mg}$ romosozumab monthly, as compared to the comparator, led to a higher risk of disease (odds ratio [OR], 2.98; 95\% confidence interval $(\mathrm{Cl}), 1 \cdot 18-7 \cdot 55 ; \mathrm{P}=0 \cdot 02$; Figure 1). Estimates from metaanalysis of 'cerebrovascular events' (27 events; OR, 2.15; 95\% Cl, 0.94-4.92; $\mathrm{P}=0.07$ ) and 'serious cardiovascular events' (183 events; OR $1 \cdot 21 ; 95 \% \mathrm{Cl}, 0 \cdot 90-1 \cdot 63 ; \mathrm{P}=0 \cdot 20$ ) were directionally concordant with increased vascular risk arising from romosozumab treatment. Sensitivity analyses using the Petomethod showed similar results (Figure $\mathbf{S 4}$ and Table S8 in the appendix).

\section{Expression of SOST mRNA}

Expression of SOST across 53 human tissue types in the Genotype-Tissue Expression consortium was highest in arterial tissue (Figure S5 in the appendix; bone expression data not available). The minor alleles of rs7209826 (G-allele) and rs188810925 (A-allele) were both associated with lower expression of SOST mRNA in various human tissues, with the strongest associations with SOST expression for each variant identified in tibial artery ( $r$ 7209826: $\left.P=1 \cdot 4 \times 10^{-8}\right)$ and aorta ( $r$ 188810925; $P=7 \cdot 6 \times 10^{-6}$; Figure S6 in the appendix).

\section{Association of rs7209826 and rs188810925 with BMD}

The minor alleles of both SNPs were associated with higher estimated heel-bone BMD (eBMD) in UKBB (rs7209826: $0.04 \mathrm{~g} / \mathrm{cm}^{2}\left[95 \% \mathrm{Cl}, 0.04-0.05 ; P=2 \cdot 3 \times 10^{-36}\right]$ per $\mathrm{G}$ allele and $\mathrm{rs} 188810925: 0.07 \mathrm{~g} / \mathrm{cm}^{2}[95 \%$ $\mathrm{Cl}, 0 \cdot 05-0 \cdot 08 ; \mathrm{P}=1 \cdot 3 \times 10^{-26}$ ] per A allele, Figure $\mathbf{S} 7$ in the appendix), and with higher LS-BMD (rs7209826: 
$0.008 \mathrm{~g} / \mathrm{cm}^{2}\left[95 \% \mathrm{Cl}, 0.005-0.01 ; \mathrm{P}=5.4 \times 10^{-07}\right]$ per G allele; rs $188810925: 0.016 \mathrm{~g} / \mathrm{cm}^{2}$ [95\% Cl, 0.01 $0.022 ; P=4.3 \times 10^{-07}$ ] per $A$ allele, Figure $\mathbf{S} 7$ in the appendix). We subsequently scaled all further genetic estimates to the increase in lumbar spine BMD seen with 12 months of $210 \mathrm{mg}$ romosozumab monthly (equivalent to a $0.09 \mathrm{~g} / \mathrm{cm}^{2}$ higher BMD). ${ }^{35}$

\section{Association with osteoporosis and fracture risk}

Scaled to match the effect of 12 months of $210 \mathrm{mg}$ romosozumab monthly on lumbar spine BMD, metaanalysis of rs7209826 and rs 188810925 yielded a $57 \%$ lower risk of osteoporosis (OR, $0.43 ; 95 \% \mathrm{CI}, 0.36$ $\left.0.52 ; \mathrm{P}=2 \cdot 4 \times 10^{-18}\right)$ and a $41 \%$ lower risk of sustaining a bone fracture $(\mathrm{OR}, 0.59 ; 95 \% \mathrm{Cl}, 0.54-0.66$; $P=1 \cdot 4 \times 10^{-24}$; Figure 2 and Figure S8 in the appendix). Associations were consistent across fracture sites (Figure $\mathbf{S 9}$ in the appendix).

\section{Association with coronary heart disease}

In meta-analysis of scaled estimates including up to 69,649 cases, the BMD-increasing SOST variants associated with an $18 \%$ higher risk of myocardial infarction and/or coronary revascularisation (OR, 1.18; 95\% Cl, 1.06-1.32; P=0.003; Figure 3 and Figure $\mathbf{S} 10$ in the appendix). Using a broader definition of CHD, which included self-reported angina and chronic stable ischemic heart disease (up to 106,329 cases), the SOST variants associated with a $13 \%$ increased risk of disease $(\mathrm{OR}, 1 \cdot 10 ; 95 \% \mathrm{Cl}, 1 \cdot 00-1 \cdot 20 ; \mathrm{P}=0 \cdot 04$; Figure 3 and Figure S10 in the appendix).

\section{Association with additional cardiometabolic risk factors and diseases}

Evaluation of cardiometabolic risk factors and diseases revealed that the BMD-increasing SOST variants associated with higher risks of hypertension (OR, 1.12; 95\% Cl, 1.05-1.20; $\left.\mathrm{P}=8 \cdot 9 \times 10^{-4}\right)$ and T2D (OR, 1.15; 95\% Cl, 1.05-1.27; P=0.003; Figure 4A).

Consistent with the effect on hypertension, we found the SOST variants to be associated with $1.3 \mathrm{mmHg}$ higher SBP $\left(95 \% \mathrm{Cl}, 0 \cdot 8-1 \cdot 9 ; \mathrm{P}=5 \cdot 9 \times 10^{-6}\right)$, but observed no effect on DBP (Figure 4B). In addition, the SOST variants associated with 0.05 standard deviation (SD) units higher WHR adjusted for $\mathrm{BMI}(95 \% \mathrm{Cl}$, 
0.02-0.08; $\left.P=8 \cdot 5 \times 10^{-4}\right)$, and, nominally, with higher serum triglycerides $(9 \cdot 6 \mathrm{mg} / \mathrm{dl}$ higher; $95 \% \mathrm{Cl}, 1 \cdot 3-18 \cdot 8$ $\mathrm{mg} / \mathrm{dl} ; \mathrm{P}=0.02$ ). We found no further associations (Figures S11-14 and Tables S9-10 in the appendix).

\section{Trans-ethnic replication}

We attempted trans-ethnic replication in the China Kadoorie Biobank. In 21,547 individuals with eBMD measurements available, the rs7209826 variant showed no evidence of association (beta=0.0016 g/ $/ \mathrm{cm}^{2}$; $\mathrm{SE}=0.001 ; \mathrm{P}=0.14)$ and strong evidence of heterogeneity with the corresponding eBMD estimate in UKBB (P-heterogeneity=1.35 $\times 10^{-28}$; Figure $\mathbf{S} 15$ in the appendix). A regional analysis showed no evidence of a suitable genetic instrument (Figure S16 in the appendix). Of note, the lack of association with CHD and other endpoints (Table S11 in the appendix) in CKB suggests that the associations obtained in Europeans were not due to pleiotropy.

\section{Triangulation of randomised controlled trials and human genetics}

We performed fixed-effect meta-analysis of 337 clinical fractures in 11,273 individuals from two RCTs with fracture outcome data (FRAME ${ }^{12}$ and $\mathrm{ARCH}^{13}$, Table S12 in the appendix), and identified that 210mg romosozumab monthly, as compared to comparator, led to a $32 \%$ lower risk of clinical fracture (hazard ratio $0.68 ; 95 \% \mathrm{Cl}, 0.55-0.85 ; \mathrm{P}=6 \cdot 0 \times 10^{-4}$; Figure 5 and Figure $\mathrm{S} 17$ in the appendix) at 12 months. This compared well to the scaled genetic estimate, using the two SOST variants, of a $41 \%$ lower risk of fracture (OR, $\left.0.59,95 \% \mathrm{Cl}, 0.54-0.66, \mathrm{P}=1.4 \times 10^{-24}\right)$. Evidence from treatment trials (two romosozumab phase III RCTs ${ }^{13,14}$ ), and human genetics (two SOST variants) showed that inhibition of sclerostin increases risk of coronary events (Figure 5), indicating that this adverse effect is likely real and target-mediated. 


\section{Discussion}

Using data from both naturally-occurring human genetics and randomised interventional trials, we have shown that while raising BMD through lifelong lowering of sclerostin is associated with lower risk of osteoporosis and fractures, it also results in a higher risk of CHD. While prior phase III RCTs of sclerostin inhibition by romosozumab suggested an increased risk of adverse cardiac events, it remained possible that the finding was due to chance owing to the low number of events (only 25 cases). Our genetic analysis, including up to 106,329 CHD cases, shows that this excess risk of CHD from sclerostin inhibition is very likely to be real. Our findings also suggest that the excess risk of coronary events may be driven by an increase in central adiposity, SBP (and hypertension), and increased risk of T2D.

There are several additional lines of evidence supporting the findings of our study. Sclerostin is expressed in cardiovascular tissues, ${ }^{33,38}$ supporting a potential biological role in these tissue types. In observational studies, higher levels of circulating sclerostin are associated with a higher risk of cardiovascular disease together with higher levels of cardiometabolic risk factors such as hypertension, T2D and central adiposity. ${ }^{39-44}$ However, further evidence has suggested that sclerostin may be up-regulated in the vasculature in response to vascular calcification, as part of a regulatory process aimed at counteracting such calcification. ${ }^{17}$ Recent murine studies have implicated sclerostin in adipocyte metabolism ${ }^{45}$ and the development of atherosclerosis, aortic aneurysm and hemopericardium. ${ }^{34,46}$ However, extrapolating findings from animal models of disease to humans is plagued by failures of translation 47,48 , and observational studies of humans can be influenced by sources of error: this is why studies employing a randomised design in humans provide more reliable evidence on causation. ${ }^{49}$

Elucidation of whether adverse effects are on- or off-target is critical ${ }^{8}$ as on-target effects would mean that any drug under development in the same class (i.e., a sclerostin inhibitor), would be expected to share a similar adverse effect profile (i.e. higher risk of vascular events). Our genetic data provide strong evidence in support of target-mediated adverse effects of sclerostin inhibition on coronary events. Sclerostin exerts its effects on bone as an inhibitor of the Wnt-signaling pathway (a pathway also previously linked to vascular calcification ${ }^{50-52}$ ) by binding to the Wnt co-receptor low density lipoprotein receptor-related protein (LRP) 5 
and $6 . .^{53}$ Protein-coding mutations in both LRP5 and LRP6 have been linked to alterations in BMD and cardiometabolic risk profiles, including insulin resistance, dyslipidemia, hypertension and CHD, ${ }^{54,55}$ which supports our findings of altered cardiometabolic disease in carriers of SOST variants.

Concerns of cardiovascular safety have also plagued other anti-osteoporotic agents. Odanacatib, a cathepsin $\mathrm{K}$ inhibitor developed by Merck for the treatment of osteoporosis, while shown to reduce fracture risk in phase III trials, was not developed further owing to an increased risk of stroke. ${ }^{56}$ While we did not identify strong associations of SOST with risk of stroke subtypes in our analyses, the point estimates for both ischemic and hemorrhagic stroke were $O R>1$. With genetic lowering of sclerostin leading to elevations in systolic blood pressure and WHR adjusted for BMI (each of which plays a causal role in stroke ${ }^{57,58}$ ), it is plausible that additional stroke cases would identify such an association. More broadly, genetically elevated BMD may exert a modest causal effect on risk of T2D and CHD. ${ }^{59}$ Interestingly, other BMD-raising therapies, e.g., denosumab or bisphosphonates, have not shown an association with increased cardiovascular risk in clinical trials. ${ }^{60-62}$

The approach to exploiting human genetics to validate target-mediated effects is well-established. ${ }^{22-26}$ In our study, we selected variants associated with reduced expression of SOST and increased BMD as proxies for the effect of sclerostin inhibition. We validated the effects of the SOST genetic variants on risk of osteoporosis and bone fracture (including fracture across several sites), and leveraged a large number of $\mathrm{CHD}$ cases (a more than 2,700-fold increase over the number of cases reported in phase III trials of romosozumab $\left.{ }^{13,14}\right)$. Our approach also facilitated identification of potential mechanisms and mediators that may drive this excess coronary risk. For example, the association of SOST variants with central adiposity, SBP and T2D has not been previously reported and if not measured in a clinical trial, such associations cannot be readily explored. Whilst trans-ethnic replication in a large East Asian biobank (CKB) could not be reliably performed owing to a lack of association of SOST variants

with BMD, we note the lack of association with CHD and other outcomes in CKB (Table S11 in the appendix). This provides an opportune means to illustrate that the multiple cardiometabolic risk factor and 
disease associations identified in the European datasets, where genetic variants in SOST do act as valid instruments for inhibition of sclerostin, did not arise as a consequence of horizontal pleiotropy (in other words, the genetic associations did not arise through a mechanism other than that which operates through inhibition of sclerostin). ${ }^{63}$

Our findings warrant a rigorous assessment of the effect of romosozumab (and other sclerostin inhibitors in clinical development) on cardiovascular disease and cardiometabolic risk factors, particularly in light of the ongoing licensing applications for this drug. Given the high short-term mortality associated with some types of fragility fracture (e.g., up to $25 \%$ mortality in the 12 months following a hip fracture ${ }^{64}$ ), a risk-benefit assessment is warranted, weighing the merits of lower fracture (and fracture-related morbidity and mortality) against the potential harm from higher risk of metabolic and vascular disease.

In conclusion, we have shown that genetic variants in the SOST locus that mimic pharmacological inhibition of sclerostin are associated with lower SOST expression, lower risk of osteoporosis and fractures but an elevated risk of coronary heart disease, likely explained by increases in systolic blood pressure, central adiposity and risk of type 2 diabetes mellitus. This adds valuable information as to whether pharmacological inhibition of sclerostin should be pursued as a therapeutic strategy for the prevention of fracture. 


\section{Declaration of Interests}

BMN is a member of the Scientific Advisory Board for Deep Genomics, a consultant for Camp4 Therapeutics Corporation, a consultant for Merck \& Co., a consultant for Takeda Pharmaceutical, and a consultant for Avanir Pharmaceuticals, Inc. MVH has collaborated with Boehringer Ingelheim in research, and in accordance with the policy of the Clinical Trial Service Unit and Epidemiological Studies Unit (University of Oxford), did not accept any personal payment. All other authors declare no competing interests.

\section{Acknowledgements}

JB is supported by funding from the Rhodes Trust, Clarendon Fund and the Medical Sciences Doctoral Training Centre, University of Oxford. JCC is funded by the Oxford Medical Research Council Doctoral Training Partnership (Oxford MRC DTP) and the Nuffield Department of Clinical Medicine, University of Oxford. TF is supported by the NIHR Biomedical Research Centre, Oxford. SLP has a Veni Fellowship (016.186.071; ZonMW) from the Dutch Organization for Scientific Research, Nederlandse Organisatie voor Wetenschappelijk Onderzoek (NWO). LM Is supported by funding from Estonian Research Council Grants PRG184, IUT20-60, IUT34-4, IUT34-11, IUT24-6. B.M.N. is supported by NIH project 5P50HD028138-27

- Project 2: Common Complex Trait Genetics of Reproductive Phenotypes. RM Is supported by funding from Estonian Research Council Grants PRG184, IUT20-60, IUT34-4, IUT34-11, IUT24-6. CML is supported by the Li Ka Shing Foundation; WT-SSI/John Fell funds; the NIHR Biomedical Research Centre, Oxford; Widenlife; and NIH (5P50HD028138-27). MVH works in a unit that receives funding from the UK Medical Research Council and is supported by a British Heart Foundation Intermediate Clinical Research Fellowship (FS/18/23/33512) and the National Institute for Health Research Oxford Biomedical Research Centre. China Kadoorie Biobank (CKB) is supported by funding from Kadoorie Charitable Foundation in Hong Kong; the UK Wellcome Trust (grant numbers 202922/Z/16/Z, 088158/Z/09/Z, 104085/Z/14/Z); Chinese Ministry of Science and Technology (grant number 2011BAI09B01); National Natural Science Foundation of China (Grant numbers 81390540, 81390541, 81390544); UK Medical Research Council (grant numbers MC_PC_13049, MC_PC_14135); GlaxoSmithKline; BHF Centre of Research Excellence, Oxford (grant number RE/13/1/30181); British Heart Foundation; and Cancer Research UK. CKB wishes to 
bioRxiv preprint doi: https://doi.org/10.1101/531004; this version posted February 1, 2019. The copyright holder for this preprint (which was not certified by peer review) is the author/funder, who has granted bioRxiv a license to display the preprint in perpetuity. It is made available under aCC-BY 4.0 International license.

thank the CKB participants; CKB project staff based at Beijing, Oxford and the 10 regional centres; the China National Centre for Disease Control and Prevention (CDC) and its regional offices for assisting with the fieldwork, and the assistance of BGI (Shenzhen, China) for conducting DNA extraction and genotyping. 


\section{References}

1 Compston JE, McClung MR, Leslie WD. Osteoporosis. Lancet 2019; 393: 364-76.

2 Black DM, Rosen CJ. Clinical Practice. Postmenopausal Osteoporosis. N Engl J Med 2016; 374: 25462.

3 Khosla S, Hofbauer LC. Osteoporosis treatment: recent developments and ongoing challenges. Lancet Diabetes Endocrinol 2017; 5: 898-907.

4 de Villiers TJ. The quest for new drugs to prevent osteoporosis-related fractures. Climacteric 2017; 20: 103-6.

5 McClung MR. Clinical utility of anti-sclerostin antibodies. Bone 2017; 96: 3-7.

6 Brunkow ME, Gardner JC, Van Ness J, et al. Bone Dysplasia Sclerosteosis Results from Loss of the SOST Gene Product, a Novel Cystine Knot-Containing Protein. Am J Hum Genet 2001; 68: 577-89.

7 Loots GG, Kneissel M, Keller $\mathrm{H}$, et al. Genomic deletion of a long-range bone enhancer misregulates sclerostin in Van Buchem disease. Genome Res 2005; 15: 928-35.

8 Rosen CJ. Romosozumab - Promising or Practice Changing? N Engl J Med 2017; 377: 1479-80.

9 Recker RR, Benson CT, Matsumoto T, et al. A randomized, double-blind phase 2 clinical trial of blosozumab, a sclerostin antibody, in postmenopausal women with low bone mineral density. $J$ Bone Miner Res 2015; 30: 216-24.

10 Reid IR. Targeting Sclerostin in Postmenopausal Osteoporosis: Focus on Romosozumab and Blosozumab. BioDrugs 2017; 31: 289-97.

11 McClung MR, Grauer A, Boonen S, et al. Romosozumab in Postmenopausal Women with Low Bone Mineral Density. N Engl J Med 2014; 370: 412-20.

12 Cosman F, Crittenden DB, Adachi JD, et al. Romosozumab Treatment in Postmenopausal Women with Osteoporosis. N Engl J Med 2016; 375: 1532-43.

13 Saag KG, Petersen J, Brandi ML, et al. Romosozumab or Alendronate for Fracture Prevention in Women with Osteoporosis. N Engl J Med 2017; 377: 1417-27.

14 Lewiecki EM, Blicharski T, Goemaere S, et al. A Phase 3 Randomized Placebo-controlled Trial to Evaluate Efficacy and Safety of Romosozumab in Men With Osteoporosis. J Clin Endocrinol Metab 2018; 103: 3183-93.

15 Evenepoel P, D'Haese P, Brandenburg V. Romosozumab in postmenopausal women with osteopenia. N Engl J Med 2014; 370: 1664.

16 Tsourdi E, Rachner TD, Hofbauer LC. Romosozumab versus Alendronate and Fracture Risk in Women with Osteoporosis. N Engl J Med 2018; 378: 195.

17 Brandenburg VM, Verhulst A, Babler A, D'Haese PC, Evenepoel P, Kaesler N. Sclerostin in chronic kidney disease-mineral bone disorder think first before you block it! Nephrol Dial Transplant 2018; gfy129.

18 EVENITY romosozumab Receives Approval In Japan For The Treatment Of Osteoporosis In Patients At High Risk Of Fracture. https://www.amgen.com/media/news-releases/2019/01/evenityromosozumab-receives-approval-in-japan-for-the-treatment-of-osteoporosis-in-patients-at-high-riskof-fracture/ (accessed Jan 21, 2019). 
19 Amgen And UCB Resubmit Biologics License Application BLA For EVENITY (romosozumab) To The US FDA. 2018; published online July 12. https://www.amgen.com/media/newsreleases/2018/07/amgen-and-ucb-resubmit-biologics-license-application-bla-for-evenityromosozumab-to-the-us-fda/ (accessed Aug 10, 2018).

20 European Medicines Agency Accepts Filing For EVENITY (Romosozumab). 2018; published online Jan 7. https://www.amgen.com/en-au/media/news-releases/2018/01/european-medicines-agencyaccepts-filing-for-evenity-romosozumab/ (accessed Aug 10, 2018).

21 Walker VM, Davey Smith G, Davies NM, Martin RM. Mendelian randomization: a novel approach for the prediction of adverse drug events and drug repurposing opportunities. Int J Epidemiol 2017; 46: 2078-89.

22 Holmes MV, Simon T, Exeter HJ, et al. Secretory phospholipase A(2)-IIA and cardiovascular disease: a mendelian randomization study. J Am Coll Cardiol 2013; 62: 1966-76.

23 Myocardial Infarction Genetics Consortium Investigators, Stitziel NO, Won $\mathrm{H}-\mathrm{H}$, et al. Inactivating mutations in NPC1L1 and protection from coronary heart disease. N Engl J Med 2014; 371: 2072-82.

24 Scott RA, Freitag DF, Li L, et al. A genomic approach to therapeutic target validation identifies a glucose-lowering GLP1R variant protective for coronary heart disease. Sci Transl Med 2016; 8: 341 ra76.

25 Ference BA, Robinson JG, Brook RD, et al. Variation in PCSK9 and HMGCR and Risk of Cardiovascular Disease and Diabetes. N Engl J Med 2016; 375: 2144-53.

26 Millwood IY, Bennett DA, Holmes MV, et al. Association of CETP Gene Variants With Risk for Vascular and Nonvascular Diseases Among Chinese Adults. JAMA Cardiol 2017; 3: 34-43.

27 Kemp JP, Morris JA, Medina-Gomez C, et al. Identification of 153 new loci associated with heel bone mineral density and functional involvement of GPC6 in osteoporosis. Nat Genet 2017; 49: 1468-75.

28 Ye W, Wang Y, Mei B, et al. Computational and functional characterization of four SNPs in the SOST locus associated with osteoporosis. Bone 2018; 108: 132-44.

29 Burgess S, Ference BA, Staley JR, et al. Association of LPA Variants With Risk of Coronary Disease and the Implications for Lipoprotein(a)-Lowering Therapies: A Mendelian Randomization Analysis. JAMA Cardiol 2018; 3: 619-27.

30 Swerdlow DI, Preiss D, Kuchenbaecker KB, et al. HMG-coenzyme A reductase inhibition, type 2 diabetes, and bodyweight: evidence from genetic analysis and randomised trials. Lancet 2015; 385: 351-61.

31 Zheng H-F, Forgetta $\mathrm{V}$, Hsu Y-H, et al. Whole-genome sequencing identifies EN1 as a determinant of bone density and fracture. Nature 2015; 526: 112-7.

32 GTEx Consortium, Laboratory, Data Analysis \&Coordinating Center (LDACC)_Analysis Working Group, Statistical Methods groups-Analysis Working Group, et al. Genetic effects on gene expression across human tissues. Nature 2017; 550: 204-13.

33 Brandenburg VM, Kramann R, Koos R, et al. Relationship between sclerostin and cardiovascular calcification in hemodialysis patients: a cross-sectional study. BMC Nephrol 2013; 14: 219.

34 Krishna SM, Seto S-W, Jose RJ, et al. Wnt Signaling Pathway Inhibitor Sclerostin Inhibits Angiotensin II-Induced Aortic Aneurysm and Atherosclerosis. Arterioscler Thromb Vasc Biol 2017; 37: 553-66.

35 Ishibashi $\mathrm{H}$, Crittenden DB, Miyauchi $\mathrm{A}$, et al. Romosozumab increases bone mineral density in postmenopausal Japanese women with osteoporosis: A phase 2 study. Bone 2017; 103: 209-15. 
36 Efthimiou O. Practical guide to the meta-analysis of rare events. Evid Based Ment Health 2018; 21: 72-6.

37 Bradburn MJ, Deeks JJ, Berlin JA, Russell Localio A. Much ado about nothing: a comparison of the performance of meta-analytical methods with rare events. Stat Med 2007; 26: 53-77.

38 Zhu D, Mackenzie NCW, Millán JL, Farquharson C, MacRae VE. The appearance and modulation of osteocyte marker expression during calcification of vascular smooth muscle cells. PLoS One 2011; 6: e19595.

39 Claes KJ, Viaene L, Heye S, Meijers B, d'Haese P, Evenepoel P. Sclerostin: Another vascular calcification inhibitor? J Clin Endocrinol Metab 2013; 98: 3221-8.

40 Novo-Rodríguez C, García-Fontana B, Luna-Del Castillo JDD, et al. Circulating levels of sclerostin are associated with cardiovascular mortality. PLoS One 2018; 13: e0199504.

41 Chang Y-C, Hsu B-G, Liou H-H, Lee C-J, Wang J-H. Serum levels of sclerostin as a potential biomarker in central arterial stiffness among hypertensive patients. BMC Cardiovasc Disord 2018; 18: 214.

42 Mathold K, Wanby P, Brudin L, Von SP, Carlsson M. Alterations in bone turnover markers in patients with noncardio-embolic ischemic stroke. PLoS One 2018; 13: e0207348.

43 Saadeldin MK, Elshaer SS, Emara IA, Maged M, Abdel-Aziz AK. Serum sclerostin and irisin as predictive markers for atherosclerosis in Egyptian type II diabetic female patients: A case control study. PLoS One 2018; 13: e0206761.

44 Albassam RS, Sabico S, Alnaami AM, et al. Bone metabolism markers are associated with neck circumference in adult Arab women. Osteoporos Int 2019; published online Jan 6. DOI:10.1007/s00198-018-04830-6.

45 Kim SP, Frey JL, Li Z, et al. Sclerostin influences body composition by regulating catabolic and anabolic metabolism in adipocytes. Proc Natl Acad Sci U S A 2017; 114: E11238-47.

46 Javaheri B, Herbert E, Hopkinson M, Al-Jazzar A, Pitsillides AA. Sost haploinsufficiency provokes peracute lethal cardiac tamponade without rescuing the osteopenia in a mouse model of excess glucocorticoids. Am J Pathol 2019; published online Jan 18. DOI:10.1016/j.ajpath.2018.12.007.

47 van der Worp HB, Howells DW, Sena ES, et al. Can animal models of disease reliably inform human studies? PLoS Med 2010; 7: e1000245.

48 Pound $\mathrm{P}$, Bracken MB. Is animal research sufficiently evidence based to be a cornerstone of biomedical research? BMJ 2014; 348: g3387.

49 Davies NM, Holmes MV, Davey Smith G. Reading Mendelian randomisation studies: a guide, glossary, and checklist for clinicians. BMJ 2018; 362: k601.

50 Wojczynski MK, Li M, Bielak LF, et al. Genetics of coronary artery calcification among African Americans, a meta-analysis. BMC Med Genet 2013; 14: 75.

51 Boström KI, Rajamannan NM, Towler DA. The regulation of valvular and vascular sclerosis by osteogenic morphogens. Circ Res 2011; 109: 564-77.

52 Touw WA, Ueland T, Bollerslev J, et al. Association of Circulating Wnt Antagonists With Severe Abdominal Aortic Calcification in Elderly Women. J Endocr Soc 2017; 1: 26-38.

$53 \mathrm{Li} \mathrm{X}$, Zhang Y, Kang H, et al. Sclerostin binds to LRP5/6 and antagonizes canonical Wnt signaling. $J$ Biol Chem 2005; 280: 19883-7. 
54 Mani A, Radhakrishnan J, Wang H, et al. LRP6 mutation in a family with early coronary disease and metabolic risk factors. Science 2007; 315: 1278-82.

55 Saarinen A, Saukkonen T, Kivelä T, et al. Low density lipoprotein receptor-related protein 5 (LRP5) mutations and osteoporosis, impaired glucose metabolism and hypercholesterolaemia. Clin Endocrinol 2010; 72: 481-8.

56 Drake MT, Clarke BL, Oursler MJ, Khosla S. Cathepsin K Inhibitors for Osteoporosis: Biology, Potential Clinical Utility, and Lessons Learned. Endocr Rev 2017; 38: 325-50.

57 Clarke R, Li L, Bennett D, et al. Abstract 16532: Impact of Systolic Blood Pressure on Cardiovascular Disease in a Chinese Population: A Mendelian Randomization Study. Circulation 2014; published online Nov 25. https://www.ahajournals.org/doi/abs/10.1161/circ.130.suppl_2.16532 (accessed Jan 24, 2019).

58 Dale CE, Fatemifar G, Palmer TM, et al. Causal Associations of Adiposity and Body Fat Distribution With Coronary Heart Disease, Stroke Subtypes, and Type 2 Diabetes Mellitus: A Mendelian Randomization Analysis. Circulation 2017; 135: 2373-88.

59 Gan W, Clarke RJ, Mahajan A, et al. Bone mineral density and risk of type 2 diabetes and coronary heart disease: A Mendelian randomization study. Wellcome Open Res 2017; 2: 68.

60 Samelson EJ, Miller PD, Christiansen C, et al. RANKL inhibition with denosumab does not influence 3-year progression of aortic calcification or incidence of adverse cardiovascular events in postmenopausal women with osteoporosis and high cardiovascular risk. J Bone Miner Res 2014; 29: 450-7.

61 Kim DH, Rogers JR, Fulchino LA, Kim CA, Solomon DH, Kim SC. Bisphosphonates and risk of cardiovascular events: a meta-analysis. PLoS One 2015; 10: e0122646.

62 Kranenburg G, Bartstra JW, Weijmans M, et al. Bisphosphonates for cardiovascular risk reduction: A systematic review and meta-analysis. Atherosclerosis 2016; 252: 106-15.

63 Holmes MV, Ala-Korpela M, Smith GD. Mendelian randomization in cardiometabolic disease: challenges in evaluating causality. Nat Rev Cardiol 2017; 14: 577.

64 Lyles KW, Colón-Emeric CS, Magaziner JS, et al. Zoledronic acid and clinical fractures and mortality after hip fracture. N Engl J Med 2007; 357: 1799-809. 
Figure 1. Meta-analysis of romosozumab and risk of cardiovascular events from phase III randomised controlled trials.

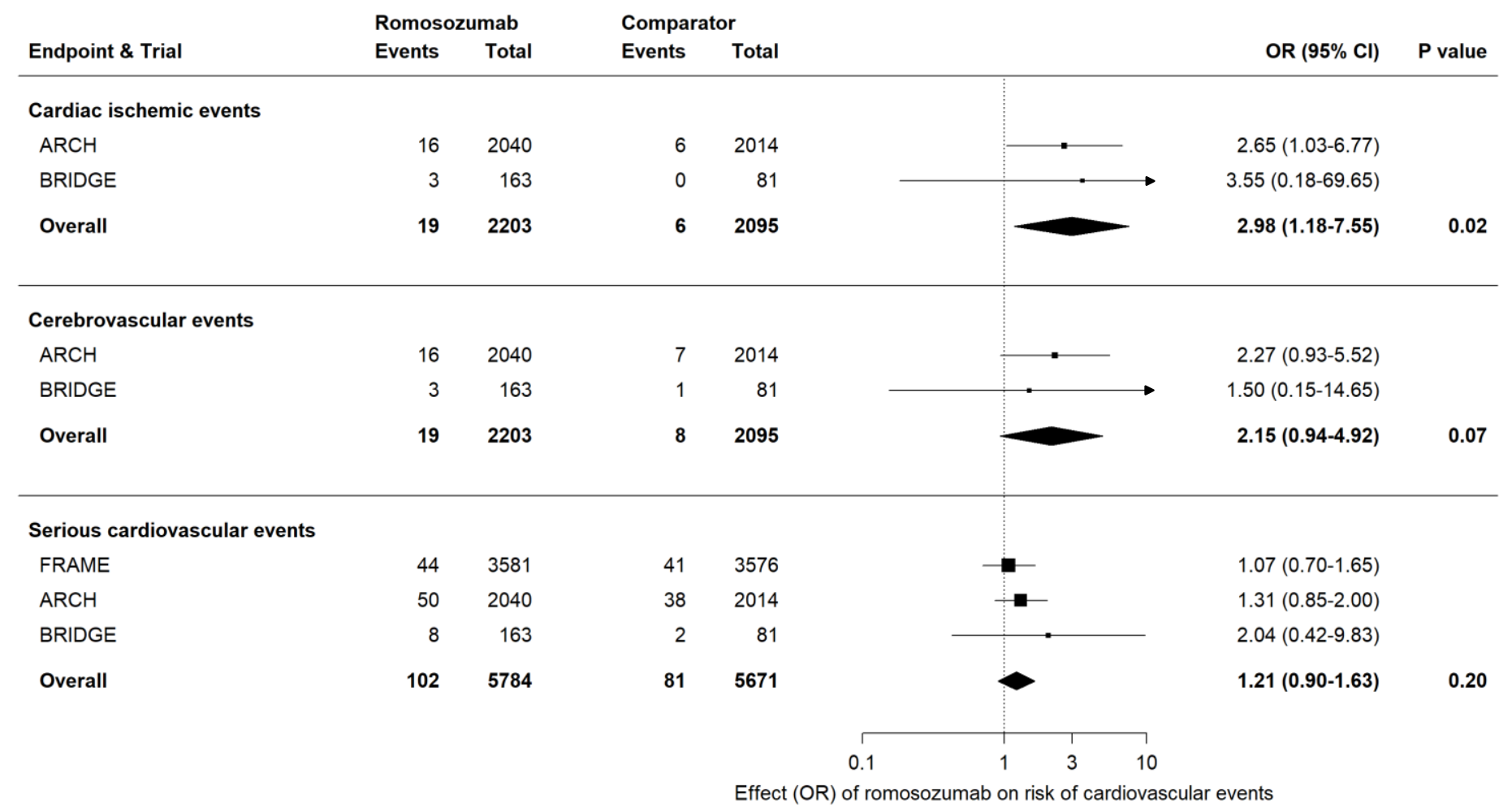

Events represents number of events adjudicated per arm, during initial 12-month double-blind period in each trial. The romosozumab-group received $210 \mathrm{mg}$ romosozumab monthly in all trials; comparator-group received placebo (FRAME and BRIDGE trials) or alendronate (ARCH). Estimates derived using the MantelHaenszel method. Outcome data for cardiac ischemic events and cerebrovascular events were only available for the ARCH and BRIDGE trials. Boxes represent point estimates of effects. Lines represent 95\% confidence intervals. OR, odds ratio; $\mathrm{Cl}$, confidence interval. 
Figure 2. Scaled estimates and meta-analysis of BMD-increasing SOST variants with risk of osteoporosis (15,329 cases) and fracture $(53,074$ cases $)$.

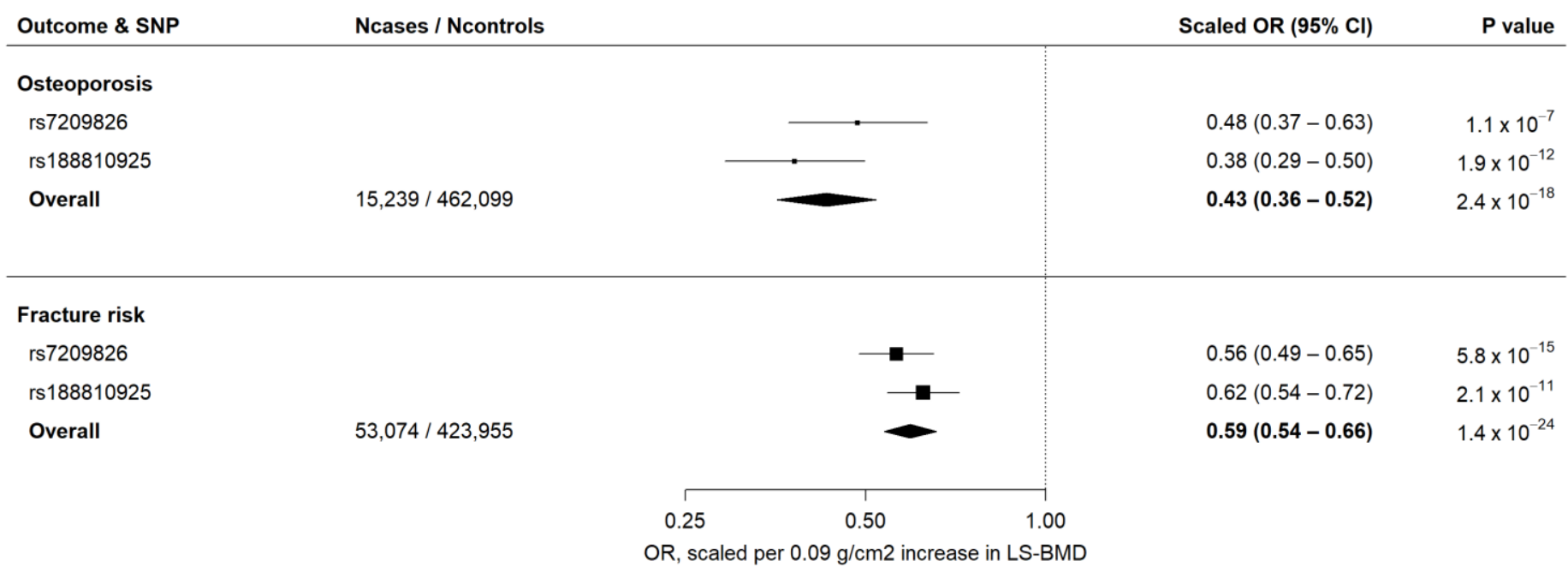

Estimates are scaled to match the effect of $210 \mathrm{mg}$ romosozumab monthly for 12 months on lumbar spine bone mineral density $\left(0.09 \mathrm{~g} / \mathrm{cm}^{2}\right.$; see Methods) and aligned to the BMD-increasing alleles. See Methods sections for outcome definitions. Boxes represent point estimates of effects. Lines represent 95\% confidence intervals. OR, odds ratio; $\mathrm{Cl}$, confidence interval; LS-BMD, lumbar spine bone mineral density. 
Figure 3. Scaled estimates and meta-analysis of BMD-increasing SOST variants with risk of myocardial infarction and/or coronary revascularisation (up to 69,649 cases) and coronary heart disease (up to 106,329 cases).

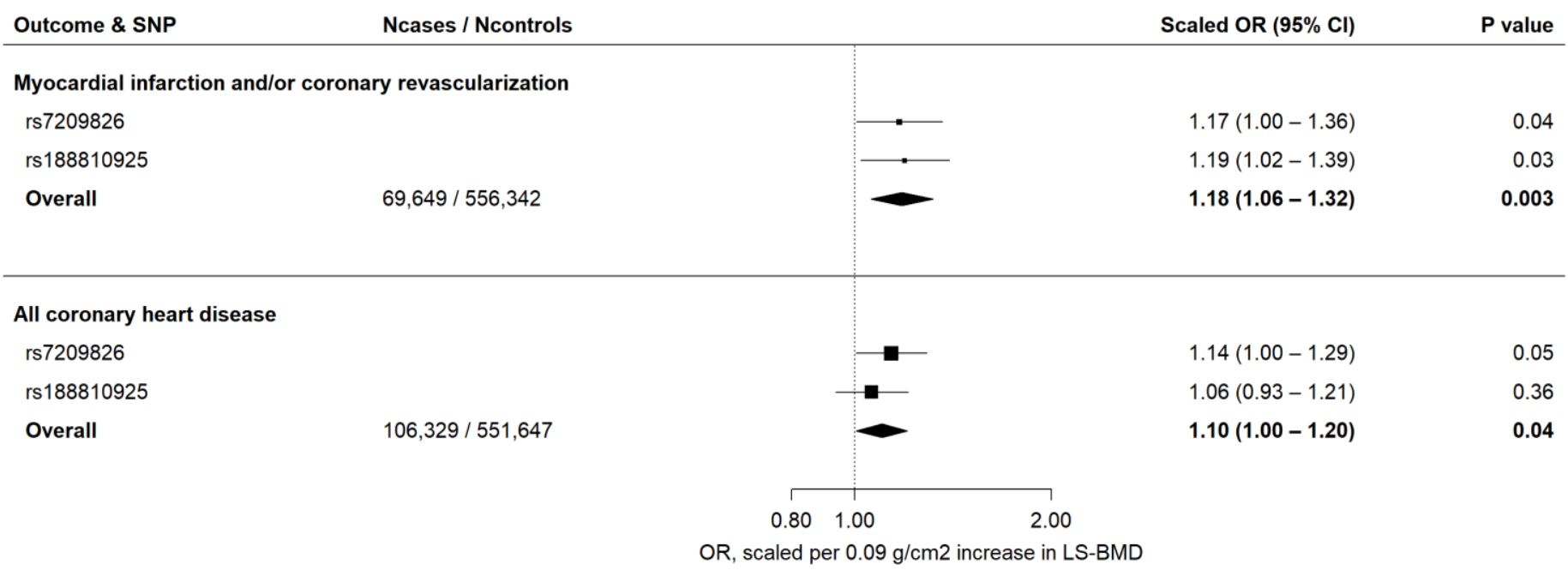

Estimates are scaled to match the effect of $210 \mathrm{mg}$ romosozumab monthly for 12 months on lumbar spine bone mineral density $\left(0.09 \mathrm{~g} / \mathrm{cm}^{2}\right.$; see Methods) and aligned to the BMD-increasing alleles. Boxes represent point estimates of effects. Lines represent 95\% confidence intervals. OR, odds ratio; $\mathrm{Cl}$, confidence interval. 
Figure 4. Meta-analysis of BMD-increasing SOST variants and cardiometabolic risk factors.

A

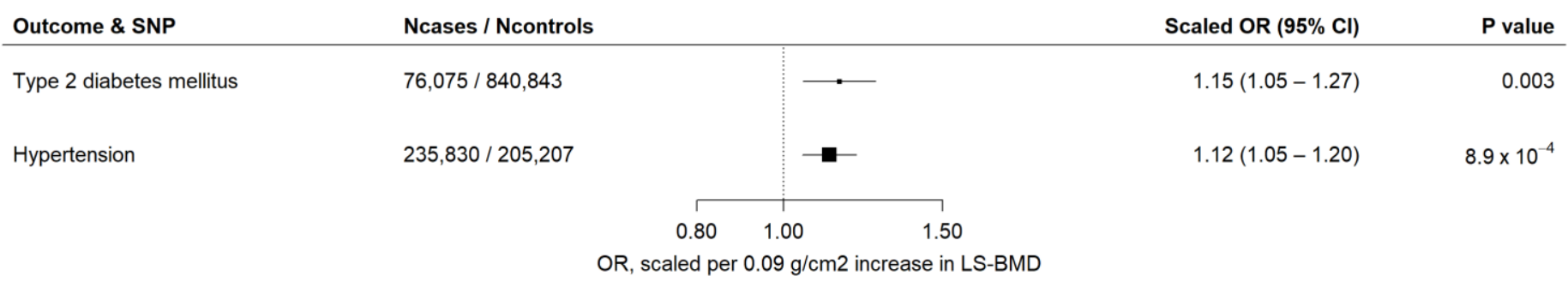

B

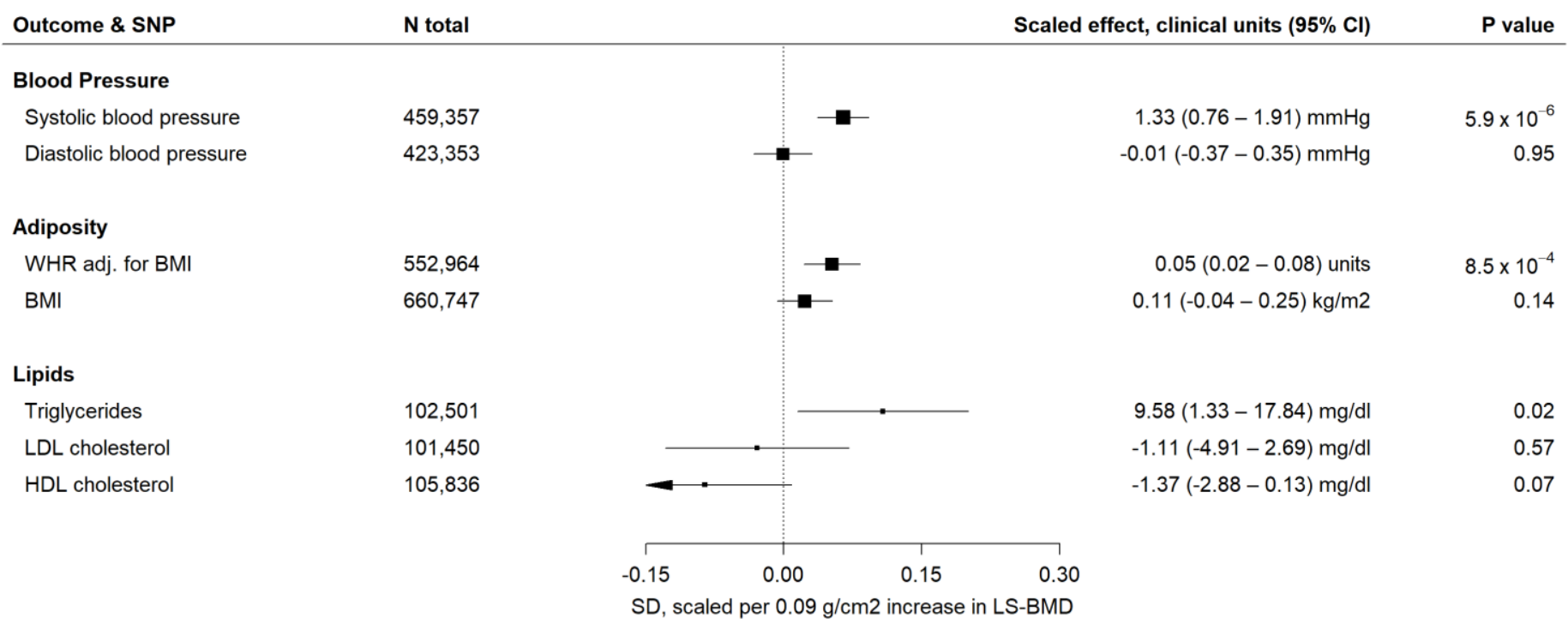

Panel A shows association with hypertension and type 2 diabetes mellitus; Panel B shows association with quantitative traits plotted in SD units, with clinical units for each trait indicated in the column on the right. All estimates are scaled to match the effect of $210 \mathrm{mg}$ romosozumab monthly for 12 months on lumbar spine bone mineral density $\left(0.09 \mathrm{~g} / \mathrm{cm}^{2}\right.$; see Methods) and aligned to the BMD-increasing alleles. The significance threshold was set at 0.0045 (see Methods for details). Boxes represent point estimates of effects in odds ratio (panel A) or standard deviation (panel B) units. Lines represent $95 \%$ confidence intervals. UKBB, UK Biobank; OR, odds ratio; $\mathrm{Cl}$, confidence interval; mmHg, millimetres of mercury; SD, standard deviations, WHR, waist to hip ratio; adj, adjusted; BMI, body mass index; LDL, low-density lipoprotein; HDL, high-density lipoprotein. 
Figure 5. Inhibition of sclerostin and risk of fracture and coronary events derived from metaanalysis of phase III randomised controlled trials of romosozumab and human genetics.

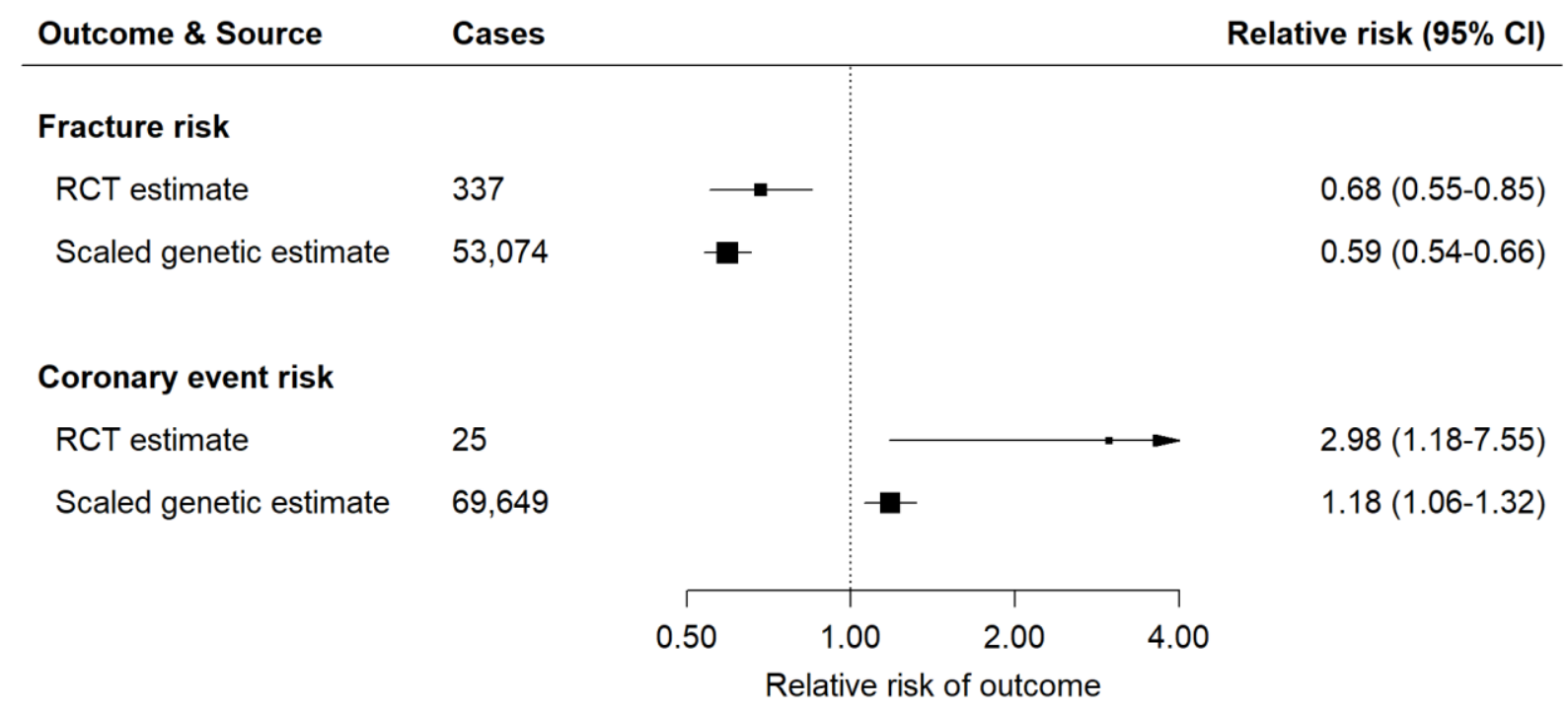

Fracture risk RCT estimate represents inverse variance weighted fixed-effect meta-analysis of estimates for hazard ratio of 'clinical fracture' at 12 months (a composite of non-vertebral or symptomatic vertebral fracture) in the $\mathrm{ARCH}$ and FRAME trials. Coronary event risk RCT estimate represents fixed-effect metaanalysis (using the Mantel-Haenszel method) of estimates for odds ratio of 'cardiac ischemic events' in the $\mathrm{ARCH}$ and BRIDGE trials. Scaled genetic estimates for fracture risk and coronary event risk represent inverse variance weighted fixed-effect meta-analyses of the scaled allelic estimates for the odds ratio of fracture risk and myocardial infarction and/or coronary revascularisation, respectively. All RCT estimates refer to the effect of romosozumab $210 \mathrm{mg}$ monthly for 12 months relative to comparator, and all genetic estimates are scaled to match the effect of $210 \mathrm{mg}$ romosozumab monthly for 12 months on lumbar spine bone mineral density $\left(0.09 \mathrm{~g} / \mathrm{cm}^{2}\right.$ increase). Boxes represent point estimates of effects. Lines represent $95 \%$ confidence intervals. $\mathrm{RR}$, relative risk; $\mathrm{Cl}$, confidence interval. 